Case Report

\title{
Management of Cerebellar Tonsillar Herniation following Lumbar Puncture in Idiopathic Intracranial Hypertension
}

\author{
Kenneth R. Hoffman, ${ }^{1}$ Sean W. Chan, ${ }^{1}$ Andrew R. Hughes, ${ }^{2}$ and Stephen J. Halcrow ${ }^{3}$ \\ ${ }^{1}$ Intensive Care Unit, Canberra Hospital, Yamba Drive, Canberra, ACT 2605, Australia \\ ${ }^{2}$ Neurology Department, Canberra Hospital, Yamba Drive, Canberra, ACT 2605, Australia \\ ${ }^{3}$ Neurosurgical Unit, Canberra Hospital, Yamba Drive, Canberra, ACT 2605, Australia
}

Correspondence should be addressed to Kenneth R. Hoffman; kenneth.hoffman@act.gov.au

Received 24 September 2014; Revised 27 December 2014; Accepted 28 December 2014

Academic Editor: Moritoki Egi

Copyright (C) 2015 Kenneth R. Hoffman et al. This is an open access article distributed under the Creative Commons Attribution License, which permits unrestricted use, distribution, and reproduction in any medium, provided the original work is properly cited.

Lumbar puncture is performed routinely for diagnostic and therapeutic purposes in idiopathic intracranial hypertension, despite lumbar puncture being classically contraindicated in the setting of raised intracranial pressure. We report the case of a 30 year-old female with known idiopathic intracranial hypertension who had cerebellar tonsillar herniation following therapeutic lumbar puncture. Management followed guidelines regarding treatment of traumatic intracranial hypertension, including rescue decompressive craniectomy. We hypothesize that the changes in brain compliance that are thought to occur in the setting of idiopathic intracranial hypertension are protective against further neuronal injury due to axonal stretch following decompressive craniectomy.

\section{Introduction}

Idiopathic intracranial hypertension (IIH) causes symptoms and signs of raised intracranial pressure (ICP) without focal neurological signs. It has an overall incidence of 1.6/100,000 per annum [1], an unclear pathogenesis, although it has significantly higher incidence in obese females aged 20-44 at 19/100,000 [2].

Lumbar puncture (LP) is classically contraindicated in the setting of raised ICP due to the risk of cerebellar tonsillar herniation. However, LP is routinely performed both for diagnostic and therapeutic purposes in IIH [3]. It has been postulated that the reason herniation does not occur in this setting is a reduction in brain compliance due to persistently high ICP [4].

To date two case reports of herniation have been reported in IIH following LP, although both of these patients had focal neurological signs on presentation and a fatal outcome [5].

We report a case of herniation following LP in a patient with IIH, her subsequent intensive care management, and clinical course.

\section{Case Report}

A 30-year-old female with a BMI of 37.9 was diagnosed with IIH seven months prior to this presentation. Her MRI at diagnosis demonstrated $4 \mathrm{~mm}$ of cerebellar tonsillar descent (Figure 1) with flattening of the posterior globes, mild distension of the optic nerve sheaths, and a partially empty sella turcica. At the time of diagnosis, her LP revealed an opening pressure of $42 \mathrm{~cm} \mathrm{H}_{2} \mathrm{O}$. She was prescribed regular oral acetazolamide and regularly underwent therapeutic drainage of $15 \mathrm{~mL}$ of cerebrospinal fluid (CSF) for symptom control without complication. Her opening pressures were consistently between 35 and $50 \mathrm{~cm} \mathrm{H}_{2} \mathrm{O}$. MR venogram demonstrated an aplastic left transverse sinus with a right transverse sinus stenosis with extrinsic compression, for which she was being considered for a stenting procedure (Figure 2).

She presented to the emergency department with four days of severe headache, nausea, and vomiting. Her past history included renal tubular acidosis diagnosed two years prior and treated with sodium bicarbonate and electrolyte 


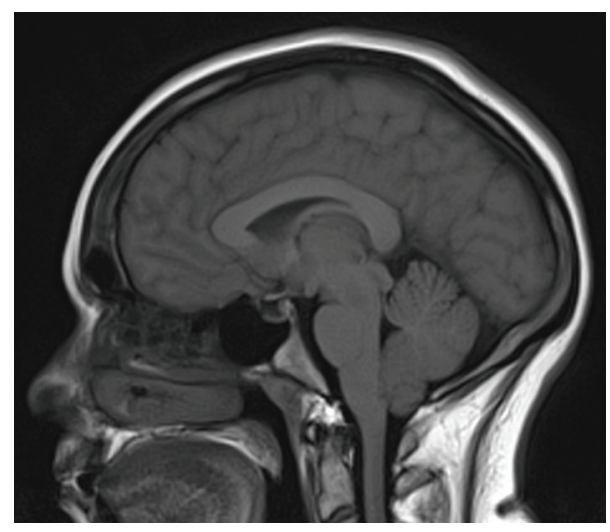

FIGURE 1: Sagittal MRI at diagnosis demonstrated $4 \mathrm{~mm}$ of cerebellar tonsillar descent.

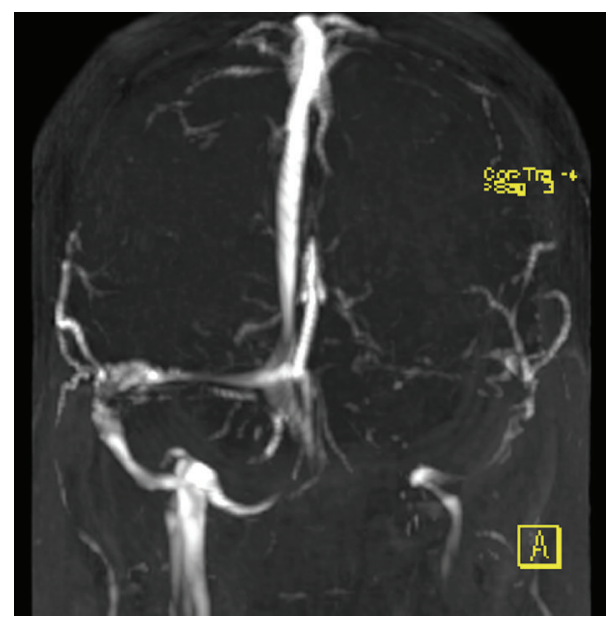

FIGURE 2: Time of flight MR venogram (anterior view) demonstrating aplastic $\mathrm{L}$ transverse sinus with $\mathrm{R}$ transverse sinus stenosis.

replacement. In the emergency department she had bilateral papilloedema with no new focal neurology. She was normothermic with a CRP of $2.8 \mathrm{mg} / \mathrm{L}$. A therapeutic LP was performed with an opening pressure of $43 \mathrm{~cm} \mathrm{H}_{2} \mathrm{O} .18 \mathrm{~mL}$ of clear CSF was drained and she was admitted to the neurology ward for observation and analgesia.

Overnight she had a medical emergency team (MET) call for hypotension attributed to hypovolemia due to ongoing vomiting, which was treated with intravenous fluids. She had a further MET call overnight for transient visual disturbance and reported being unable to appreciate light/dark and had severe headache. Her pupils were bilaterally dilated but responsive to light. She was alert and interactive and had no motor deficits. The visual changes resolved without intervention and she was taken for a CT venogram, which demonstrated bilateral tentorial herniation with $10 \mathrm{~mm}$ of cerebellar tonsillar herniation. There were no features of dural venous thrombosis.

She was reviewed on the morning of day 1 and found to be agitated, Glasgow Coma Scale (GCS) 13 (M6, V4, E3), with a fixed dilated right pupil $7 \mathrm{~mm}$. Her left pupil was $5 \mathrm{~mm}$

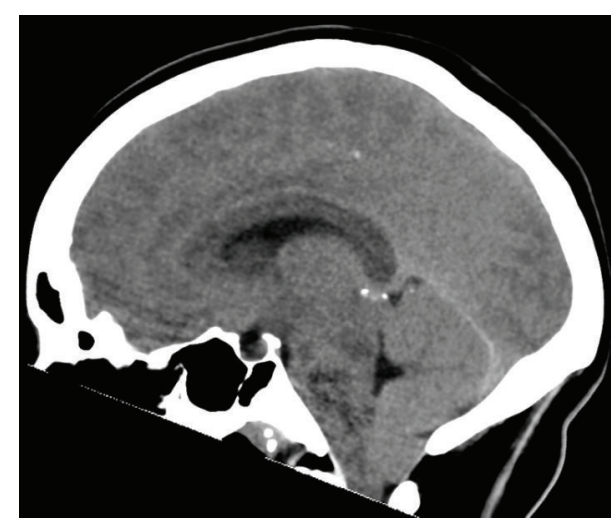

FIGURE 3: Sagittal CT scan demonstrating $10 \mathrm{~mm}$ of tonsillar descent with posterior fossa crowding.

and reactive. Plantar response was upgoing bilaterally and her neck was hyperextended with extreme pain on neck flexion. Heart rate was $65 \mathrm{bpm}$ and blood pressure was $112 / 66 \mathrm{mmHg}$. She was transferred urgently to the intensive care unit (ICU) and intubated for anticipated decline whilst awaiting a CT brain. During the CT scan she became bradycardic with a heart rate of 30 and asystolic pauses, treated with osmotic therapy of $10 \mathrm{~mL} 20 \%$ sodium chloride. Her BP was then recorded as $147 / 77 \mathrm{mmHg}$. The CT scan again showed $10 \mathrm{~mm}$ of tonsillar descent and an increase in fourth ventricle size with posterior fossa crowding (Figure 3 ). Her initial LP result showed a mildly elevated white cell count of $7 \times 10^{6} / \mathrm{L}$ (normal $<5 \times 10^{6} / \mathrm{L}$ ) and she was commenced on aciclovir empirically.

She was treated with a further $20 \mathrm{~mL}$ of $20 \%$ sodium chloride and taken for urgent insertion of an external ventricular drain (EVD), a technically difficult procedure due to small ventricular size, which rapidly improved right pupil reactivity although it remained sluggish. She returned to ICU with the EVD on free drainage $15 \mathrm{~cm}$ above the tragus. Her ICP remained persistently high at $>45 \mathrm{~cm} \mathrm{H}_{2} \mathrm{O}$ and the EVD was lowered to $10 \mathrm{~cm}$ to allow further drainage. As her pressure on previous LPs was $35-50 \mathrm{~cm} \mathrm{H}_{2} \mathrm{O}$, a target of $35 \mathrm{~cm} \mathrm{H}_{2} \mathrm{O}$ was agreed upon for initial management.

On day 2 she had persistently high ICP $>60 \mathrm{~cm} \mathrm{H}_{2} \mathrm{O}$ despite aggressive management with hyperventilation to $\mathrm{PaCO}_{2}$ of $35 \mathrm{mmHg}$, osmotic therapy with $20 \%$ sodium chloride, deep sedation with morphine and midazolam, and aggressive temperature management, targeting normothermia. Her ICP did not respond to intermittent paralysis; thus a paralytic infusion was not commenced. CT scan demonstrated $12 \mathrm{~mm}$ of tonsillar herniation. She was treated with $16 \mathrm{mg}$ dexamethasone and taken for rescue bifrontal decompressive craniectomy. The brain was noted to be grossly swollen, hyperaemic, and herniating with marked cortical venous distension. The craniectomy was insufficient alone to provide cerebral decompression due to the inelasticity of the dura; thus the latter was opened with multiple slits during which a cortical vein was lacerated requiring diathermy. The EVD was removed and a fibre-optic pressure monitor was placed. 


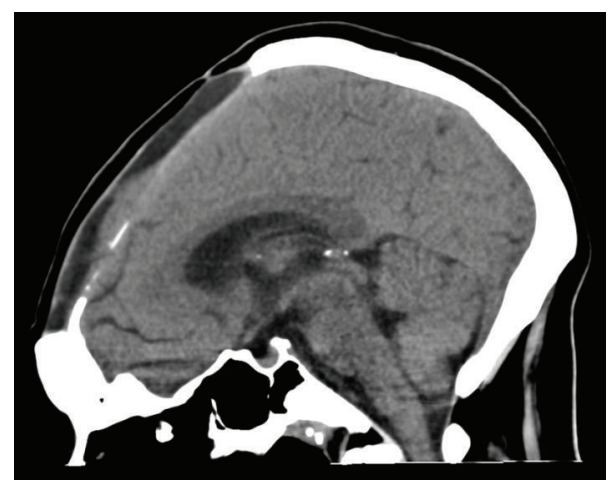

FIGURE 4: Sagittal CT demonstrating normal cerebellar tonsillar position after decompressive craniectomy.

Following this, her ICP remained $<10 \mathrm{~cm}_{2} \mathrm{O}$. Postoperative CT scan showed $8 \mathrm{~mm}$ of tonsillar herniation and a significant increase in size of the third ventricle. CSF culture was negative and viral PCR for HSV1, HSV2, enterovirus, and VZV were also negative and her empiric aciclovir was ceased.

On day 3 high dose sedation was ceased with slow recovery including transient diabetes insipidus treated with desmopressin and reintubation for poor sputum clearance. She had a gradual neurological improvement and was discharged to the neurology ward with a GCS 15 and no focal neurology on day 15 of her admission.

Unfortunately she developed CSF rhinorrhoea and pneumocephalus due to a CSF fistula through the frontal air sinus requiring surgical repair. She subsequently developed a venous infarct complicated by seizures and the requirement for intubation for reduced level of consciousness.

After rehabilitation, she was discharged home on day 69 after admission and was able to function independently, although she had not returned to work. At the time of publication she is undergoing neuropsychological testing to evaluate her readiness to return to work. Her cerebellar tonsils had returned to a normal position on a follow-up outpatient CT scan (Figure 4).

\section{Discussion}

Given the rarity of cerebellar tonsillar herniation in this setting, literature regarding management was not available. Management was thus based upon physiological principles such as the Monro-Kellie hypothesis and consensus between medical teams.

There were several unusual aspects to her presentation. Firstly, she had a known vascular malformation with an aplastic left transverse sinus and stenosis of the right transverse sinus. Vascular abnormalities do not preclude the diagnosis of IIH and have been implicated in the pathogenesis [6]. Resolution of symptoms has been reported in those with dural venous sinus stenting [7], the procedure she was being considered for. She also already had $4 \mathrm{~mm}$ of cerebellar tonsillar descent documented on an MRI six months prior to presentation; however criterion for identifying a clearly pathological Chiari 1 malformation is $5 \mathrm{~mm}$ [8]. It has previously been suggested that the combination of low lying tonsils and raised ICP potentially contributed to herniation in this setting [5]. Despite mild elevation of her CSF white cell count and empiric treatment with aciclovir, her viral PCR returned negative and her clinical presentation was not consistent with encephalitis.

Prior to her urgent transfer to ICU, there was a high index of suspicion of raised ICP given her fixed dilated pupil. However, at the time she was conscious, moving all limbs, and interactive despite severe pain. This was considered unusual compared with the significantly decreased level of consciousness associated with raised ICP in traumatic brain injury (TBI). It is therefore pertinent to recognize that TBI represents a syndrome comprising both the primary brain injury and secondary rise in ICP with hematoma formation and/or oedema.

There were several differences in this patient compared with the two previously reported cases. Firstly, herniation in IIH usually occurs as a late complication following lumbar peritoneal shunting [9]. This is consistent with the hypothesis that a reduction in pressure gradually restores normal brain compliance [4]. Secondly, in the rare case of herniation following LP the time course has previously been reported as occurring within minutes [5], whereas this case occurred over a period of 14 hours. This may suggest an alternative cause of the rise in ICP, perhaps a rebound phenomenon due to her inability to tolerate oral acetazolamide due to vomiting. Acetazolamide has been demonstrated to significantly reduce intracranial pressure [10].

In the absence of supportive literature, management followed local practice of TBI patients with raised ICP. Medical management initially involved sedation, analgesia, $\mathrm{PaCO}_{2}$ control, targeted temperature management, and intermittent osmotic therapy with $20 \%$ sodium chloride. As the above interventions were not able to control her ICP, barbiturate coma was unlikely to be successful, and a surgical option was pursued.

Issues arose however regarding the ICP target once the EVD was inserted given the fact that her usual pressures were between 35 and $50 \mathrm{~cm} \mathrm{H}_{2} \mathrm{O}$. An initial target of $35 \mathrm{~cm} \mathrm{H}_{2} \mathrm{O}$ was decided upon to provide a moderate reduction in pressure, without requiring excessive vasopressor doses to achieve an adequate cerebral perfusion pressure. The goal was to gradually reduce this down to $30 \mathrm{~cm} \mathrm{H}_{2} \mathrm{O}$.

The surgical procedure to control her critical intracranial hypertension was performed to provide rapid and life-saving control. It appeared that the aplastic left transverse sinus and consequent anomalous posterior cranial fossa drainage led to grossly hypertrophied cerebellar and circular venous sinuses. Foramen magnum decompression and cerebellar tonsillar disimpaction were considered in addition to bifrontal craniectomy. However, these were not performed acutely because her aberrant venous anatomy presented an unacceptably high risk of uncontrollable venous sinus haemorrhage.

Decompressive craniectomy was performed as a rescue procedure. In a recent trial, worse functional outcomes have been identified in TBI patients with second line decompressive craniectomy [11]. It has been postulated that this is the result of neuronal injury due to axonal stretch with herniation 


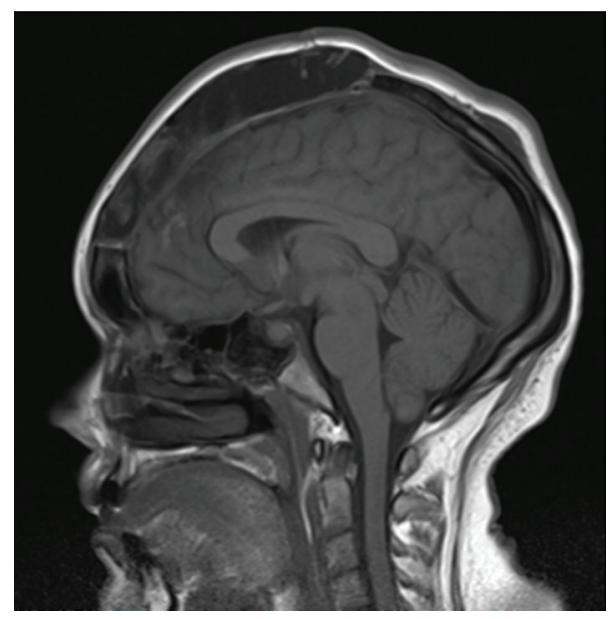

FIGURE 5: Sagittal MRI 3 weeks after decompressive craniectomy.

via the craniectomy site. Given the hypothesis regarding reduced brain compliance in $\mathrm{IIH}$ [4] it is possible that this is protective against further neuronal injury due to stretch. In this case, the decompressive craniectomy not only brought her intractable intracranial hypertension under immediate control but also resulted in disimpaction of her cerebellar tonsillar herniation (Figure 5).

\section{Conclusion}

In this patient with IIH who had cerebellar tonsillar herniation following LP, management similar to traumatic intracranial hypertension was successful. ICP targets in this setting are unknown; however we recommend a moderate reduction compared with baseline pressures as long as focal neurology resolves. Given the rapid improvement in ICP control and favourable neurological outcome, we recommend that decompressive craniectomy be considered, especially given the possible protection against further brain injury from axonal stretch due to reduced brain compliance.

\section{Consent}

The patient has provided informed consent for the case report to be published.

\section{Conflict of Interests}

The authors declare there is no conflict of interests regarding publication of this paper.

\section{References}

[1] B. E. McGeeney and D. I. Friedmanet, "Pseudotumor Cerebri pathophysiology," Headache: The Journal of Head and Face Pain, vol. 54, no. 3, pp. 445-458, 2014.

[2] F. J. Durcan, J. J. Corbett, and M. Wall, "The incidence of pseudotumor cerebri. Population studies in Iowa and Louisiana," Archives of Neurology, vol. 45, no. 8, pp. 875-877, 1988.
[3] S. R. A. Paruchuri, M. Lawlor, K. Kleinhomer, L. Mason, and C. Johnson, "Risk of cerebellar tonsillar herniation after diagnostic lumbar puncture in pseudotumor cerebri," Anesthesia and Analgesia, vol. 77, no. 2, pp. 403-404, 1993.

[4] M. Salman, "Why does tonsillar herniation not occur in idiopathic intracranial hypertension?" Medical Hypotheses, vol. 53, no. 4, pp. 270-271, 1999.

[5] H. C. Sullivan, "Fatal tonsillar herniation in pseudotumor cerebri," Neurology, vol. 41, no. 7, pp. 1142-1144, 1991.

[6] B. R. Wakerly, M. H. Tan, and E. Y. Ting, "Idiopathic intracranial hypertension," Cephalgia, 2014.

[7] R. M. Ahmed, M. Wilkinson, G. D. Parker et al., "Transverse sinus stenting for idiopathic intracranial hypertension: a review of 52 patients and of model predictions," The American Journal of Neuroradiology, vol. 32, no. 8, pp. 1408-1414, 2011.

[8] A. O. Aboulezz, K. Sartor, C. A. Geyer, and M. H. Gado, "Position of cerebellar tonsils in the normal population and in patients with Chiari malformation: a quantitative approach with MR imaging," Journal of Computer Assisted Tomography, vol. 9, no. 6, pp. 1033-1036, 1985.

[9] F. J. Rowe, "Diplopia and visual impairment as presenting symptoms of shunt failure in association with tonsillar herniation in idiopathic intracranial hypertension," Strabismus, vol. 20, no. 4, pp. 181-184, 2012.

[10] M. R. Chaaban, E. Illing, K. O. Riley, and B. A. Woodworth, "Acetazolamide for high intracranial pressure cerebrospinal fluid leaks," International Forum of Allergy and Rhinology, vol. 3, no. 9, pp. 718-721, 2013.

[11] D. James Cooper, J. V. Rosenfeld, L. Murray et al., "Decompressive craniectomy in diffuse traumatic brain injury," The New England Journal of Medicine, vol. 364, no. 16, pp. 1493-1502, 2011. 


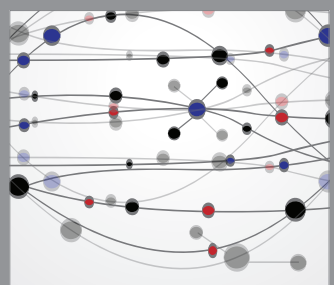

The Scientific World Journal
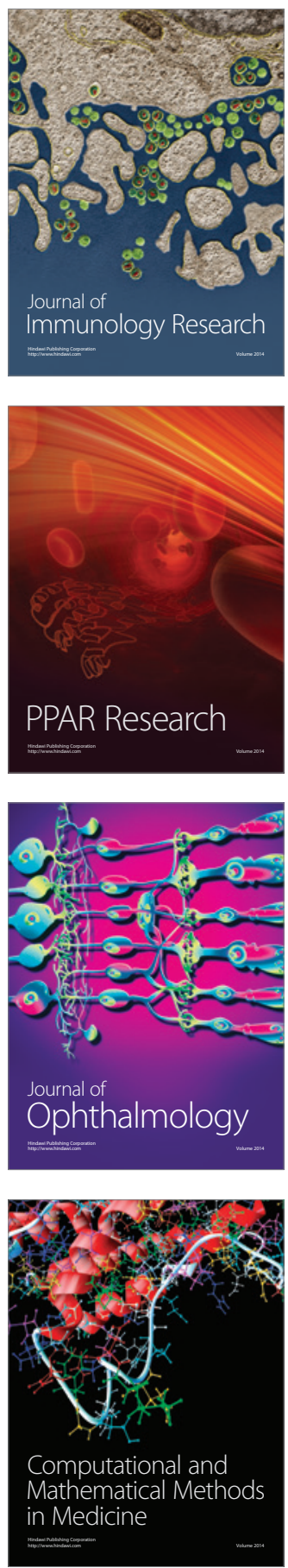

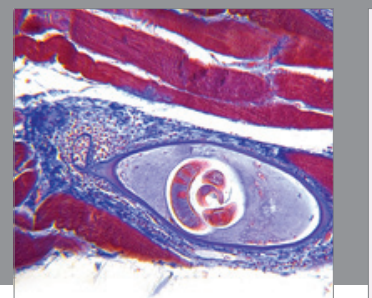

Gastroenterology

Research and Practice
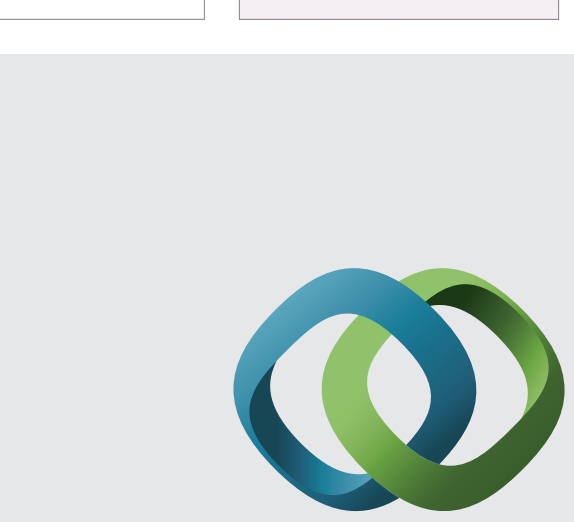

\section{Hindawi}

Submit your manuscripts at

http://www.hindawi.com
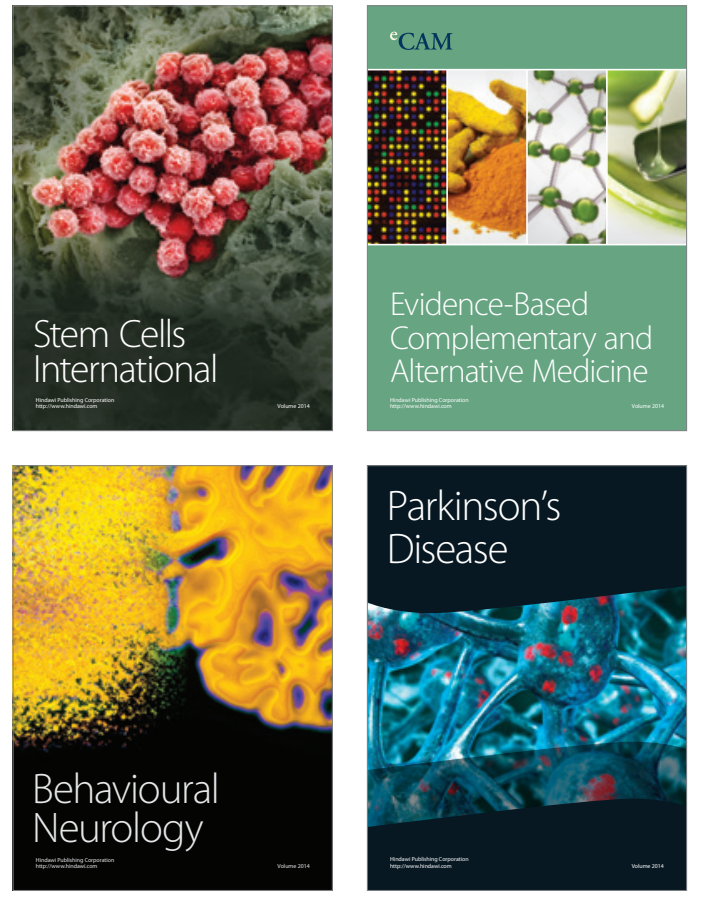
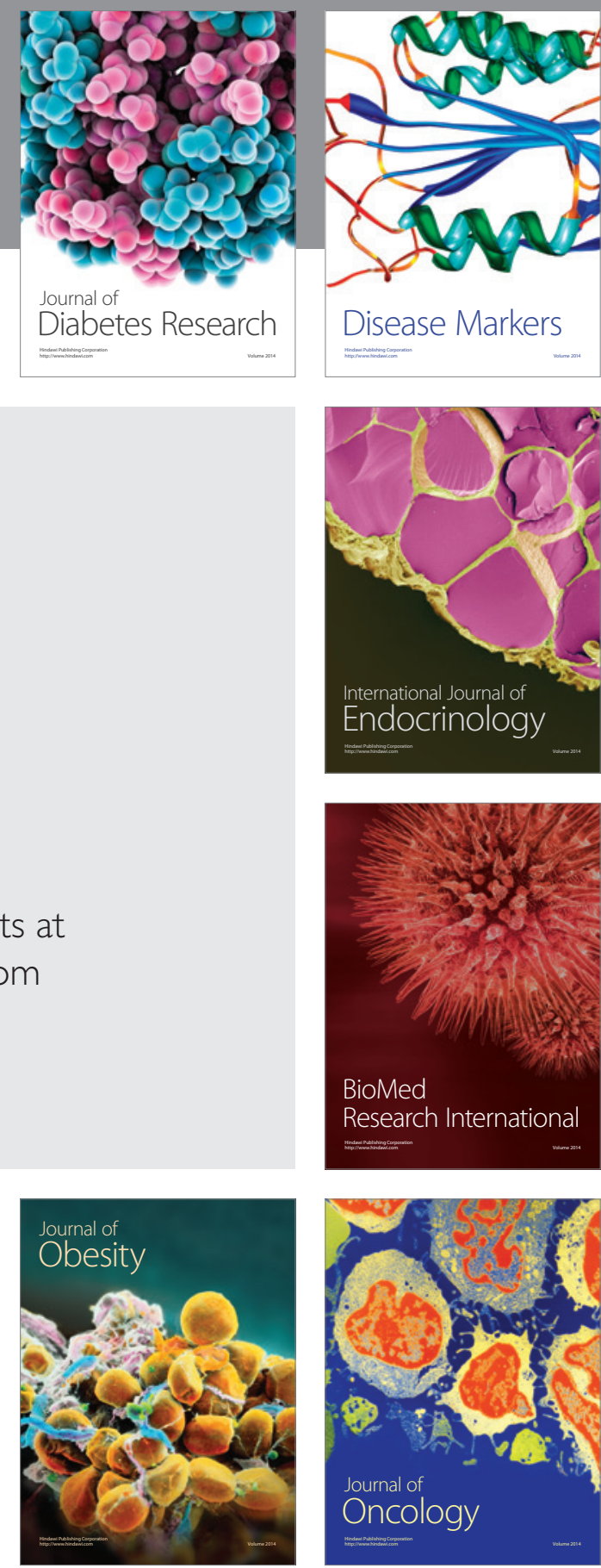

Disease Markers
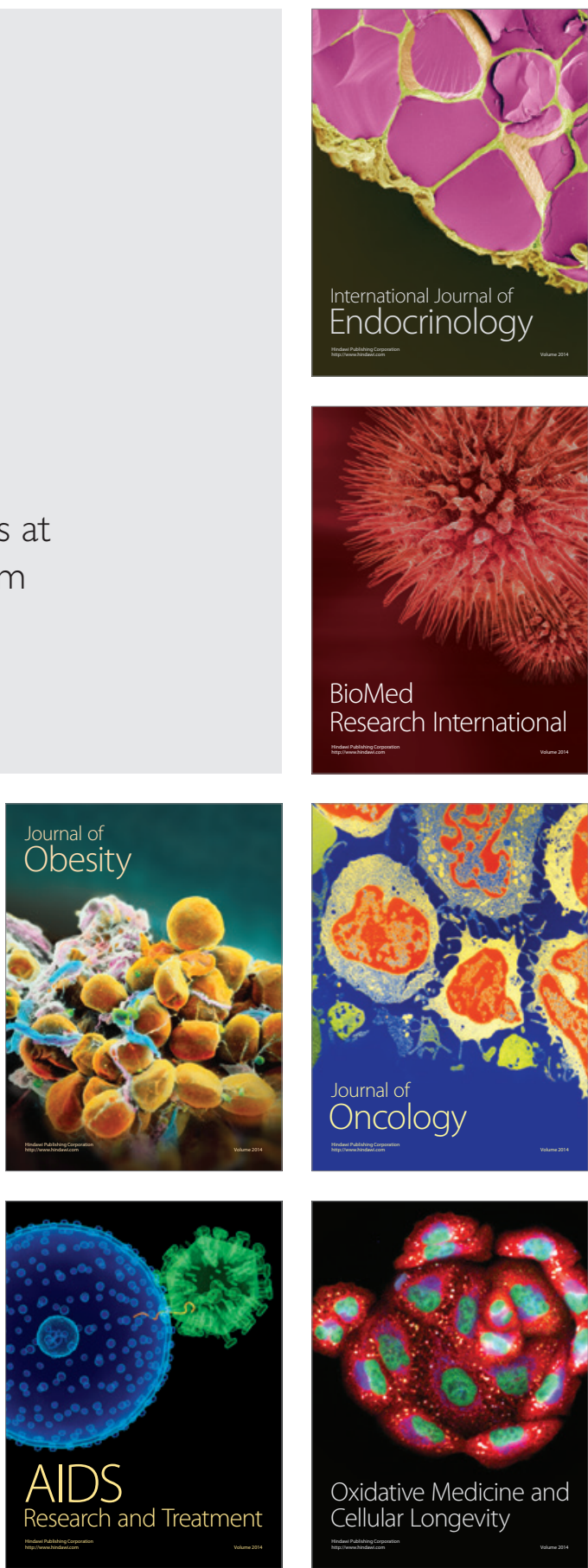